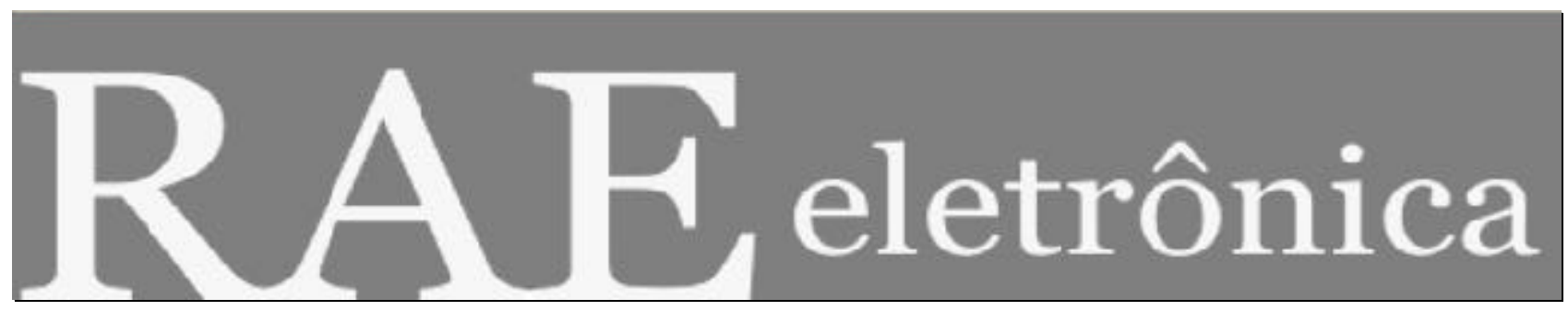

\title{
APRENDIZAGEM GERENCIAL: TEORIA E PRÁTICA
}

Por:

\author{
Liege Viviane dos Santos de Moraes \\ Maria Aparecida da Silva \\ Cristiano J. C. A. Cunha
}

RAE-eletrônica, v. 3, n. 1, Art. 7, jan./jun. 2004

http://www.rae.com.br/eletronica/index.cfm?FuseAction=Artigo\&ID=1854\&Secao=ORGANIZA \&Vol ume $=3 \&$ Numero $=1 \&$ Ano $=2004$

CCopyright, 2004, RAE-eletrônica. Todos os direitos, inclusive de tradução, são reservados. É permitido citar parte de artigos sem autorização prévia desde que seja identificada a fonte. A reprodução total de artigos é proibida. Os artigos só devem ser usados para uso pessoal e nãocomercial. Em caso de dúvidas, consulte a redação: redacao@ rae.com.br.

A RAE-eletrônica é a revista on-line da FGV-EAESP, totalmente aberta e criada com o objetivo de agilizar a veiculação de trabalhos inéditos. Lançada em janeiro de 2002, com perfil acadêmico, é dedicada a professores, pesquisadores e estudantes. Para mais informações consulte o site www.rae.com.br/eletronica.

RAE-eletrônica

ISSN 1676-5648

(C)2004 Editora: Fundação Getulio Vargas - Escola de Administração de Empresas de São Paulo

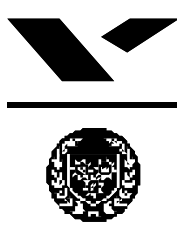

F U N D A G Ã O GETUUO VARGAS

Escola de Adniriistraña de Empresas de Stro Panulo 


\title{
APRENDIZAGEM GERENCIAL: TEORIA E PRÁTICA
}

\section{RESUMO}

A literatura indica um crescente interesse por uma área emergente de pesquisa e prática no campo das organizações: a aprendizagem gerencial. Tal área está preocupada com a investigação dos processos de aprendizagem que contribuem para a prática da gestão, o que inclui, embora não exclusivamente, a educação e o desenvolvimento gerencial. Tendo em vista que, no Brasil, esta área de pesquisa é ainda incipiente, o propósito deste artigo é apresentar uma revisão da literatura que consolida a aprendizagem gerencial como uma área emergente de pesquisa e prática no campo das organizações.

\begin{abstract}
The literature indicates an increasing interest in an emergent arena of research and practice in the field of organizations: management learning. This area investigates the learning process that contributes to the practice of management, which also includes management education and development, though not exclusively. Inasmuch in Brazil this area is incipient, the purpose of this paper is to present a review of the literature that consolidate management learning as a new arena of theory and practice in the field of organizations.
\end{abstract}

\section{PALAVRAS-CHAVE}

Executivo, adulto, aprendizagem, aprendizagem gerencial.

\section{KEY-WORDS}

Executive, adult, learning, management learning. 


\section{INTRODUÇÃO}

Atualmente, o mundo dos negócios muda de forma rápida, freqüente e inesperada como conseqüência das forças que regem a sociedade e a economia. Neste ambiente, os executivos ${ }^{1}$ não podem mais prever o futuro numa perspectiva de longo prazo para, então, definir planos estáveis de ação. Ao invés disto, estes profissionais precisam aprender rapidamente de modo a lidar com a necessidade de mudanças súbitas que se devem, muitas vezes, a fatores adversos e imprevisíveis.

No Brasil, um dos reflexos deste clima de mudança é a disseminação de programas de educação para executivos, como os mestrados na modalidade profissional ou mestrados executivos. Em contrapartida, cresce a preocupação com a identidade destes programas e sua efetividade em garantir a aquisição de competências gerenciais atuais (Antonello e Ruas, 2002; Ruas, 2002).

Em outros países, esta preocupação é antiga e originou esforços significativos de pesquisa sobre o processo de aprendizagem gerencial, o que levou mesmo as formas mais tradicionais de desenvolvimento gerencial, como os MBA (Master in Business Administration), a buscar sistemas mais adaptáveis e efetivos na tarefa de ajudar os executivos a aprenderem (Daudelin, 1996).

Essas pesquisas consolidam a aprendizagem gerencial como uma área emergente de pesquisa e prática no campo das organizações que, como tal, preocupa-se com o estudo dos mecanismos que contribuem para a prática da gestão, onde a educação e o desenvolvimento gerencial são partes integrantes deste processo (Burgoyne e Reynolds, 1997; Fox, 1997).

Uma vez que mo Brasil as investigações nesta área são incipientes, o propósito deste artigo é apresentar uma revisão da literatura que consolida a aprendizagem gerencial como uma área emergente de pesquisa e prática no campo das organizações. Discutindo-se sobre o conceito de aprendizagem gerencial, o artigo possibilita aquecer o debate sobre a problemática da identidade e efetividade dos programas de educação para executivos (Antonello e Ruas, 2002; Ruas, 2002). Além disso, o conteúdo permite uma ampliação do entendimento sobre os processos individuais de aprendizagem que são necessários, embora não suficientes, para a aprendizagem no nível organizacional (Ruas, 2001b; Elkjaer, 2001; Richter, 1998).

$\mathrm{O}$ artigo foi estruturado da seguinte maneira. Primeiramente, discute-se as raízes e a evolução do conceito da aprendizagem gerencial. Após isso, apresentamse as influências teóricas que têm contribuído para teoria e prática na área da aprendizagem gerencial. Em seqüência, apresenta-se uma revisão da literatura para consolidar a aprendizagem gerencial como uma nova área no campo das organizações. Esta seção expõe alguns comentários sobre as principais descobertas de pesquisas empíricas nesta área. Por fim, são tecidas algumas considerações com base nas idéias apresentadas ao longo do texto.

\section{AS RAIZES E A EVOLUÇÃO DO CONCEITO DA APRENDIZAGEM GERENCIAL}

Tradicionalmente, a aprendizagem gerencial sempre foi vista como produto da educação e do desenvolvimento gerencial formal. Nesta perspectiva, a aprendizagem é concebida como um processo formal, que ocorre em ocasiões isoladas e alheias ao dia a dia do executivo (Fox, 1997).

Esta concepção é decorrente da visão tradicional que se consolidou na área da educação ao longo do tempo, onde a aprendizagem é vista como um processo de trans ferência de conhecimento de uma fonte 
ORGANIZAÇÕES - APREDIZAGEM GERENCIAL: TEORIA E PRÁTICA

Liege Viviane dos S. de Moraes - Maria Aparecida da Silva - Cristiano J. A. C. Cunha

para o aprendiz, que irá recebê-lo e armazená-lo para uso futuro (Richter, 1998; Gherardi, Nicolini e Odella, 1998).

No campo das organizações, as críticas a esta concepção da aprendizagem decorrem da dificuldade de adaptação e aplicação prática das teorias que os programas de formação gerencial procuram ensinar (Livingston, 1971). Além disso, questiona-se se, mesmo quando aplicadas na prática, estas teorias geram atitudes que garantam uma ação gerencial efetiva (Davies e Easterby-Smith, 1984).

Acontece que os modelos de aprendizagem e ação originados com a ciência da administração são deficientes em explicar como os executivos lidam com os desafios diários do seu trabalho. A racionalidade técnica que deu base a estes modelos não fomenta as habilidades reflexivas necessárias para que os executivos tomem decisões sob a incerteza e em confronto com ambientes turbulentos ( Schön, 1983; Marsick, 1988).

Assim, embora o modelo tradicional de aprendizagem gerencial valorize apenas a educação e o desenvolvimento gerencial formal, ou seja, que ocorrem em sala de aula (Fox, 1997), aumenta a crença de que os maiores ganhos profissionais e organizacionais se originam da aprendizagem que ocorre no próprio ambiente de trabalho (Carnevale, 1986; Marsick e Watkins, 1997).

Apesar disto e da dificuldade em mensurar os ganhos reais obtidos com programas de formação gerencial, no Brasil os investimentos em treinamento e desenvolvimento continuam sendo feitos sem uma avaliação sistemática (Antonello e Ruas, 2002). É importante ressaltar que isto ocorre num momento em que há uma disseminação destes programas, por um lado, como reflexo de decisões da CAPES e, por outro, como reflexo do clima de mudança e de pressão por resultados nas organizações (Ruas, 2002).

Considerando-se a baixa experiência desses programas no Brasil, "a consolidação de sua própria identidade vai exigir uma série de avaliações e redefinições, no curto e médio prazo. [...] Dependendo da cultura, objetivos, condições e massa crítica da instituição proponente, a disposição final desses programas pode ser uma tanto diferenciada, embora atendendo o mesmo tipo de demanda" (Ruas, 2002, p.1-2).

Isto faz com que, de forma crescente, pesquisadores brasileiros demonstrem a preocupação com a "transmissão do que é aprendido em sala de aula para atividade profissional e a sustentabilidade dos efeitos e repercussões das experiências em educação executiva de curta duração" (Antonello e Ruas, 2002, p.1). Embora os programas nacionais de formação gerencial procurem inserir inovações conceituais para aprimorar a aprendizagem gerencial, elas ainda não representam uma perspectiva de desenvolvimento de competências (Ruas, 2001a).

Em outros países, o uso de recursos inovadores de aprendizagem, como a reflexão e a experiência, são fruto de pesquisas sobre o processo de aprendizagem gerencial e são consideradas "eventos críticos para o desenvolvimento de competências gerenciais específicas” (Daudelin, 1996, p. 36).

Estas pesquisas fundamentam-se em diferentes teorias sobre aprendizagem e educação de adultos, assim como em teorias sobre a aprendizagem em ambientes de trabalho. Estas duas áreas têm contribuído para a evolução do conceito da aprendizagem gerencial - o qual rompe com a visão tradicional que vê a aprendizagem como transferência de conhecimento -, bem como para a consolidação da aprendizagem gerencial como uma área emergente de pesquisa e prática no campo das organizações. 
ORGANIZAÇÕES - APREDIZAGEM GERENCIAL: TEORIA E PRÁTICA

Liege Viviane dos S. de Moraes - Maria Aparecida da Silva - Cristiano J. A. C. Cunha

Os conceitos que definem a aprendizagem gerencial como uma área emergente no campo das organizações e seus principais estudos serão apresentados de forma detalhada na seção 4 deste artigo. A seguir, descrevem-se as influências teóricas sobre tal área.

\section{INFLUÊNCIAS TEÓRICAS DA ÁREA DA APRENDIZAGEM E EDUCAÇÃO NA TEORIA E PRÁTICA DA APRENDIZAGEM GERENCIAL}

Como ficará claro na seção 4 , toda a teoria e prática na área da aprendizagem gerencial têm recebido a influência, em maior ou menor grau, de diferentes abordagens da educação e aprendizagem, especialmente no que refere-se à educação e aprendizagem de adultos.

Para que se possa compreender estas influências é preciso, antes de mais nada, conhecer as abordagens teóricas na área da educação e aprendizagem que originam essas influências. Esta seção apresenta as diferentes orientações teóricas que procuram explicar o fenômeno da aprendizagem.

\section{As abordagens teóricas na área da aprendizagem e educação}

A maioria dos psicólogos que estudavam o fenômeno da aprendizagem até 1950 definiam a aprendizagem como uma mudança no comportamento, o que é resultado de uma visão da aprendizagem como produto (Merrian e Caffarella, 1991).

A aprendizagem vista como um processo, ao invés de um produto final, implica levar em conta o que acontece quando ela ocorre. As explicações sobre este processo são chamadas de teorias de aprendizagem e, de acordo com Merriam e Caffarella (1991), podem ser classificadas de acordo com suas orientações teóricas, quais sejam: a behaviorista, a cognitivista, a humanista e a social (Quadro 1 a seguir).

A classificação feita por essas autoras indica que, embora a aprendizagem possa ser compreendida de diferentes maneiras, a "maioria das definições inclui os conceitos de mudança de comportamento e experiência" (Merriam e Caffarella, 1991, p. 124).

Conforme indica o Quadro 1, a idéia principal da abordagem behaviorista é que a aprendizagem é manifestada através de mudanças de comportamento, sendo determinada pelos elementos do ambiente na qual o sujeito se insere.

Já a abordagem cognitivista considera que o homem não pode ser considerado um ser passivo. Ele organiza suas experiências e procura lhes dar significado. Como resultado, essa abordagem enfatiza a importância dos processos mentais do processo de aprendizagem, os quais estariam sob o controle do aprendiz.

A terceira abordagem descrita no Quadro na próxima página - a humanista - assume que o potencial humano para o crescimento é ponto de partida para a compreensão do processo de aprendizagem. Considera que as pessoas podem controlar seu próprio destino, possuem liberdade para agir e que o comportamento delas é conseqüência da escolha humana. Os princípios que regem tal abordagem são a autodireção e o valor da experiência no processo de aprendizagem.

Por fim, a abordagem social descrita no quadro assume que as pessoas aprendem observando outras pessoas no interior do contexto social. Nessa abordagem a "aprendizagem é uma função da interação da pessoa, do ambiente e do comportamento" (Merriam e Caffarella, 1991, p. 139). 
ORGANIZAÇÕES - APREDIZAGEM GERENCIAL: TEORIA E PRÁTICA

Liege Viviane dos S. de Moraes - Maria Aparecida da Silva - Cristiano J. A. C. Cunha

\section{Quadro 1: Quatro Orientações de Aprendizagem.}

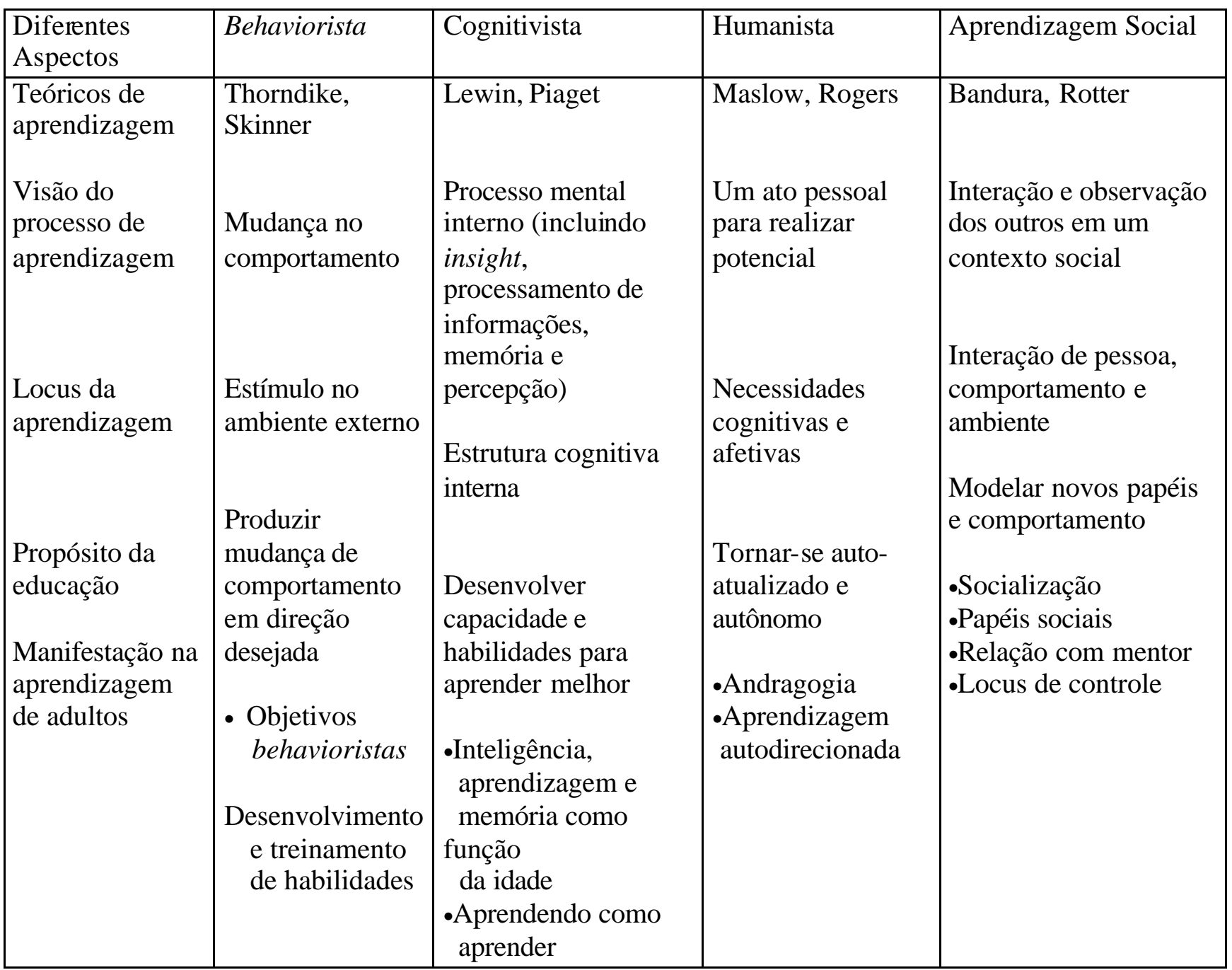

Fonte: Merriam e Caffarella (1991)

Segundo Mezirow (1996), o significado da aprendizagem para as diversas teorias depende dos pressupostos que fundamentam seus paradigmas, bem como das suposições referentes à sua natureza e função. Para esse autor, a compreensão de tais pressupostos pode levar ao entendimento das diversas vertentes de pesquisa na área. Para tanto, ele identificou três paradigmas distintos na área da educação e aprendizagem: o radicalismo ocidental, o paradigma interpretativo e o paradigma emancipatório.

No primeiro, a realidade existe independentemente das representações mentais ou lingüísticas no mundo. Sob este paradigma entende-se que o conhecimento é objetivo e não depende nem deriva de atividades subjetivas; sua lógica e a racionalidade são formais e de causa-efeito (Mezirow, 1996).

O paradigma interpretativo é marcado pela obra de Vigotsky e oferece uma nova concepção sobre aprendizagem e educação. Baseando-se no argumento de que as categorias cognitivas são sociais em origem, Vigotsky advoga que a compreensão é inerentemente social, ao invés de um ato biológico. Segundo Mezirow (1996) o paradigma interpretativo teve a contribuição de diversas abordagens de 
ORGANIZAÇÕES - APREDIZAGEM GERENCIAL: TEORIA E PRÁTICA

Liege Viviane dos S. de Moraes - Maria Aparecida da Silva - Cristiano J. A. C. Cunha

vertente humanista como a fenomenologia, a hermenêutica, o interacionismo simbólico e a etnometodologia.

Por fim, o paradigma emancipatório baseia-se na concepção de Sócrates de que existe um tipo de autoreflexão que pode libertar os indivíduos da tirania e da dependência de falsas opiniões. Fundamenta-se, ainda, na proposta de Habermas de que o entendimento e a aprendizagem se estruturam por meio da comunicação humana (Mezirow, 1996).

O desenvolvimento dessas diferentes teorias indica uma evolução no entendimento sobre a educação e aprendizagem, isto é, a descoberta de novos conceitos sobre o processo de aprendizagem e, como conseqüência, a utilização de diferentes formas de ensino. No que se refere especificamente à aprendizagem e educação de adultos, essas teorias consagram diferentes aspectos da aprendizagem na fase adulta como a natureza: autodirecionada, social e emancipatória. Estes aspectos serão exposto a seguir.

\section{A natureza autodirecionada da aprendizagem na fase adulta}

Um dos conceitos que mais tem influenciado a teoria e prática da aprendizagem gerencial é aquele que concebe a aprendizagem de adultos como um processo autodirecionado. Tal conceito decorre de uma corrente pragmática da educação de adultos que acredita que o adulto autodireciona sua aprendizagem de modo a encarar melhor as mudanças que fazem parte de sua vida diária .

Sob esta perspectiva, o componente fundamental da aprendizagem é a autodireção que determina que ela pode ocorrer sem a ajuda de um instrutor, onde o adulto "tem a responsabilidade primária pelo planejamento, execução e avaliação de suas próprias experiências de aprendizagem" (Merriam e Caffarella, 1991, p.41).

A autodireção foi inicialmente concebida por Knowles, em 1975, ao propor uma abordagem andragógica para a educação de adultos. Por muito tempo, a autodireção foi considerada um elemento central da aprendizagem de adultos (Tough, 1971; Cranton, 1996), utilizado amplamente como base para programas de treinamento e desenvolvimento gerencial (Marsick, 1988; Marsick e Watkins, 1998).

O que a autodireção procura demonstrar é que cabe ao adulto escolher os diferentes caminhos para atingir diferentes propósitos de aprendizagem. Isso gerou a idéia de que a aprendizagem de adultos pode ocorrer em duas modalidades: formal e informal. Cervero, Miller e Dimmock (1986) citam como exemplos de atividades formais de aprendizagem cursos de especialização e seminários. Como atividades informais, a leitura de livros, revistas e a consulta a especialistas.

$\mathrm{Na}$ área da aprendizagem gerencial é este conceito que sustenta a crença de que os executivos aprendem apenas através de atividades formais ou informais. Contudo, estudos nesta área têm recebido importantes contribuições de uma corrente teórica que legitima a natureza social da aprendizagem na fase adulta, como pode ser visto a seguir.

\section{A natureza social da aprendizagem na fase adulta}

Mesmo que se aceite as características individualistas que marcam a idéia da aprendizagem de adultos como um processo autodirecionado, a maior parte dessa aprendizagem depende e deriva de um contexto social particular e das experiências que este proporciona. 
ORGANIZAÇÕES - APREDIZAGEM GERENCIAL: TEORIA E PRÁTICA

Liege Viviane dos S. de Moraes - Maria Aparecida da Silva - Cristiano J. A. C. Cunha

De acordo com Candy (1991), a autodireção não deve obscurecer que a aprendizagem "ocorre em grupos antes que em isolamento completo; mesmo aqueles que começam suas aprendizagens sozinhos, procuram outros aprendizes para mensurar seus progressos e para compartilhar suas experiências" (p. $301)$.

Essa noção tem origem em teorias que se fundamentam no paradigma interpretativo da aprendizagem, também conhecido como revolução cognitiva. Assim, cognição e aprendizagem estão, "intimamente relacionadas ao contexto material, simbólico e social na qual ocorrem. Para entender as competências cognitivas e sua aquisição, é necessário explorar os contextos específicos de atividade e práticas sociais na qual eles ocorrem" (Gherardi, Nicoline e Odella, 1998, p. 275).

A aprendizagem refere-se, então, à tentativa dos adultos em se apropriar e utilizar um corpo de conhecimentos já legitimados por um grupo social, envolvendo "a aceitação de regras sociais que governam determinada área ou domínio de conhecimento” (Candy, 1991, p. 302). É neste sentido que a natureza da aprendizagem na fase adulta é dependente do contexto, ou seja, "a maioria da aprendizagem de adultos ocorre em contextos sociais e deriva de pressões sociais de vários tipos" (Candy, 1991, p. 301).

Essa perspectiva se opõe à abordagem behaviorista que é "compatível com o modelo mecânico de organização, quando o treinamento e desenvolvimento gerencial emergiram como um campo da prática" (Marsick, 1988, p. 187). Esta abordagem passou a ser questionada porque se percebeu que as práticas tradicionais de educação não fomentam as habilidades reflexivas necessárias ao trabalho na atualidade (Marsick, 1988) e que os maiores ganhos de aprendizagem ocorrem no ambiente de trabalho e não em decorrência de programas formais (Carnevale, 1986).

Embora no Brasil haja uma carência de pesquisas que revelem dados quantitativos sobre os resultados de programas de educação e treinamento (Antonello e Ruas, 2002), em outros países pesquisas indicam que "apenas $15 \%$ dos ganhos individuais podem ser atribuídos à programas de aprendizagem formais em instituições, enquanto 85\% resulta da aprendizagem no ambiente de trabalho" (Carnevale, 1986, p.24).

No que se refere especificamente aos programas de formação gerencial, prevalece a dúvida de que eles de fato ensinam aos gerentes o que eles precisam saber. Na maioria das vezes estes programas enfatizam modelos racionais de decisão, distorcendo a habilidade de aprender enquanto se ganha experiência no trabalho (Richter, 1998; Marsick, 1988; Livingston, 1971).

Disto decorre que "bons aprendizes em salas de aula várias vezes tornam se lentos aprendizes entre executivos" (Livingston, 1971, p. 79). No entender de Richter (1998), o principal aspecto negligenciado pela visão tradicional refere-se ao poder que os gerentes têm de influenciar as relações sociais dentro do trabalho e a utilização disso para aumentar seus conhecimentos e melhorar seu desempenho.

Ocorre que, a aprendizagem profissional não é exclusivamente uma atividade individual nem formal, mas que

“(...) em organizações de trabalho, pessoas e grupos criam conhecimento, negociando o significado de palavras, ações, situações e artefatos materiais [...] o conhecimento não é o que reside na cabeça de uma pessoa, em livros ou em bancos de dados. Conhecer é ser capaz de participar com os requisitos de 


\begin{abstract}
ORGANIZACÕES - APREDIZAGEM GERENCIAL: TEORIA E PRÁTICA
Liege Viviane dos S. de Moraes - Maria Aparecida da Silva - Cristiano J. A. C. Cunha

competência em uma rede complexa de relacionamentos entre pessoas e atividades" (Gherardi, Nicolline e Odella, 1998, p. 274).
\end{abstract}

Esta concepção surge de uma corrente teórica que acredita que a realidade é socialmente construída, sendo que os significados são negociados por meio da linguagem e das interações sociais (Lave e Wenger, 1991; Brown, Collins e Douguid, 1989). Nela a aprendizagem vista como uma atividade totalmente individual, principalmente em ambientes de trabalho, é uma visão limitada. Ao invés de o aprendiz ser considerado um ator que apenas processa informações, deve-se considerar que ele aprende por meio de interações sociais, dentro de determinado ambiente sociocultural.

A crença que predomina nesta perspectiva teórica é que ser detentor de determinado conhecimento não é o que leva à perícia; ao contrário, é o processo de participação em atividades sociais que "conecta o indivíduo primeiro ao relacionamento social e então, como conseqüência, às habilidades e conhecimentos da perícia prática no interior daqueles relacionamentos" (Eisenhart, 1995, p. 35).

Com base nestes conceitos, teorias como a de Lave e Wenger (1991) têm sido desenvolvidas para explicar a aprendizagem que ocorre em ambientes de trabalho. Estes autores afirmam que, nestes ambientes, a aprendizagem ocorre no interior de comunidades de prática $^{2}$, ou seja, agremiações informais definidas não só pelos seus membros, mas pela maneira compartilhada na qual eles fazem as coisas, interpretam as experiências e perpetuam uma prática.

A diferença entre estas comunidades e redes de aprendizagem ${ }^{3}$ é que, na primeira a aprendizagem evolui a partir de relações sociais espontâneas, isto é, não planejadas, que permitem ao adulto tornar-se membro de um grupo de profissionais e na segunda as relações são planejadas com o propósito explícito de aprendizagem (Richter, 1998).

É sobre este caráter não planejado da aprendizagem que Marsick e Watkins (1997) procuraram desenvolver seu modelo para facilitar a aprendizagem gerencial. Estas autoras sugerem que existe um tipo de aprendizagem que ocorre de forma acidental, ou seja, não premeditada pelo aprendiz, no dia a dia de trabalho. Esta é uma releitura das autoras da perspectiva teórica de Dewey e Lewin sobre o papel da experiência na aprendizagem que, segundo elas, tem sido mal interpretada por autores como Kolb (1976) e Munford (1997) que simplificam as idéias originais daqueles autores.

O modelo das autoras concebe que o entendimento da aprendizagem gerencial deve considerar a relação entre o contexto social, o executivo e suas experiência. Isto favorece o entendimento de dimensão fundamental da aprendizagem na fase adulta: a reflexão. Esta dimensão, que tem influenciado significativamente a teoria e prática da aprendizagem gerencial, será exposta a seguir.

\title{
A natureza emancipatória da aprendizagem na fase adulta
}

Diversas teorias procuram explicar a aprendizagem gerencial com base nas mudanças de consciência que ocorrem na fase adulta. Essas teorias baseiam-se no paradigma emancipatório da aprendizagem (Mezirow, 1996).

Entre elas, destacam-se as teorias de Freire e de Mezirow, ambas focadas nas mudanças de consciência que ocorrem na fase adulta. A teoria transformativa de Mezirow lida especificamente com o processo de aprendizagem, enquanto a de Freire é uma teoria para a educação de adultos (Merriam e Caffarella, 1991). 
ORGANIZAÇÕES - APREDIZAGEM GERENCIAL: TEORIA E PRÁTICA

Liege Viviane dos S. de Moraes - Maria Aparecida da Silva - Cristiano J. A. C. Cunha

Sob essa perspectiva, acredita-se que a forma como o homem se relaciona com o mundo designará a forma de sua consciência e de sua ação:

"Toda compreensão de algo corresponde, cedo ou tarde, a uma ação. Captado um desafio, compreendido, admitidas as hipóteses, o homem age. A natureza da ação corresponde à natureza da compreensão. Se a compreensão é crítica, a ação também será. Se é mágica a compreensão, mágica será a ação" (Freire, 1982, p.106).

Para Mezirow (1991), "a aprendizagem de adultos envolve a projeção imaginativa de modelos simbólicos de valores em nosso senso de percepções para construir o significado das experiências. Este processo imaginativo é filtrado por perspectivas de significado - conjunto de pressuposições psicoculturais aprendidas que funcionam como códigos reguladores da percepção e cognição" (p. 188).

Através da definição de nossas expectativas, as perspectivas de significado ordenam seletivamente o que e como aprendemos. Cada perspectiva contém um número de esquemas de significado, que "são conhecimentos, crenças, valores de julgamento e sentimentos particulares que se articulam em uma interpretação" (Ibid., p. 44). São estas perspectivas que sustentam nos adultos seus critérios de julgamento ou de avaliação para o certo e o errado, o belo e o feio, o mal ou o bem. Elas são modeladas por fatores sociolingüísticos, epistêmicos e psicológicos (Cranton, 1996). Freire (1982) dá um bom exemplo de como isso ocorre ao explicar como a cultura do silêncio molda a consciência do brasileiro de uma forma ingênua e mágica desde a infância.

Essa noção é especialmente importante tendo em vista o papel e influência dos executivos na sociedade moderna, pois é com base em sua consciência que o adulto age. Mas o adulto pode transformar suas perspectivas de significado e, com isso, a sua ação. Isso só é possível, pois a aprendizagem na fase adulta possui um domínio ${ }^{4}$ que lhe permite obter mudanças de consciência que podem alterar seus padrões de ação.

De acordo com La Paro (1991) essa é a área mais negligenciada por educadores e treinadores, o que é uma pena, pois através dessa capacidade o adulto pode explorar "como forças psicológicas e culturais têm sido assimiladas de forma não crítica, para vislumbrar como elas têm restringido sua ação pessoal" (p. 25).

É neste sentido que tem se criticado o uso exclusivo de abordagens baseadas no conceito de autodireção para guiar a aprendizagem gerencial. Embora muito utilizadas em programas de desenvolvimento gerencial, essas abordagens se fundamentam em perspectivas humanistas e andragógicas - como as de Carl Rogers ou Malcom Knowles - que não garantem aos executivos da capacidade de análise crítica necessária à ação gerencial na atualidade (Marsick, 1988).

De forma similar, Reynolds (1998) sugere que, embora essas abordagens sejam utilizadas como metodologias para moldar a ação gerencial, "é duvidoso que na prática elas vinculem o exame crítico de processos sociais e políticos" (p.185). Para o autor, tal exame se faz necessário tendo em vista que os executivos são responsáveis pelas formas de organizar o trabalho que podem dar origem a problemas econômicos, sociais e ecológicos.

É essa capacidade - de refletir criticamente - que leva à aprendizagem de double loop proposta por Argyris (1977), sendo que sua ausência explica as reações defensivas dos executivos em situações que deveriam mudar suas concepções e aprender. 
ORGANIZAÇÕES - APREDIZAGEM GERENCIAL: TEORIA E PRÁTICA

Liege Viviane dos S. de Moraes - Maria Aparecida da Silva - Cristiano J. A. C. Cunha

Segundo Reynolds (1998), refletir criticamente significa reconhecer os aspectos sociais, políticos e históricos da realidade que se vivencia. Considerando que a atividade gerencial é permeada por valores, crenças e pressuposições o executivo pode, por meio da reflexão crítica, questionar pressupostos culturais e psicológicos mudando sua ação. Para este autor, os executivos tornam-se criticamente reflexivos quando percebem que aquilo que fazem pode ser modificado ou melhorado. Os profissionais que atingem este nível de aprendizagem levam em conta questões morais, éticas e epistemológicas sobre o trabalho e não apenas as questões instrumentais.

A seguir, serão apresentados alguns conceitos que da aprendizagem gerencial, bem como comentários sobre estudos realizados nesta área.

\section{UMA NOVA ÁREA DE PESQUISA E PRÁTICA NO CAMPO DAS ORGANIZAÇÕES}

Os conceitos teóricos apresentados na seção anterior têm fundamentado as pesquisas sobre a aprendizagem gerencial que, por sua vez, fundamentam modernas práticas de educação e desenvolvimento gerencial.

Tendo origem com o ceticismo em relação à efetividade dos programas de educação e desenvolvimento gerencial, o crescente interesse de pesquisa sobre esta temática tem consolidando a aprendizagem gerencial como uma área emergente de pesquisa e prática.

Em países como o Reino Unido, onde a educação de executivos faz parte de uma longa tradição, a preocupação com a indústria da educação gerencial é antiga e estimulou investigações com o intuito de explorar o paradoxo existente entre o sucesso e crescimento dessa indústria e as "contínuas críticas e dúvidas sobre seus objetivos, métodos e efetividade” (Burgoyne e Reynolds, 1997, p. 7).

Sendo assim, a aprendizagem gerencial preocupa-se com a investigação dos "processos de aprendizagem que contribuem para a prática da gestão, incluindo a educação e o desenvolvimento gerencial" (Fox, 1997, p. 34-35).

Burgouyne e Reynolds (1997) afirmam que a aprendizagem gerencial "refere-se tanto ao o que é adquirido quanto ao o como essa aquisição ocorre quando indivíduos e grupos sociais adquirem a habilidade de gerenciar" (p.5). Para estes autores, a aprendizagem gerencial é uma área, tanto da prática profissional, quanto de investigação teórica, cujo intuito é diminuir as lacunas existentes entre estes dois domínios.

No campo da prática, a aprendizagem gerencial refere-se às atividades como treinamento, educação e desenvolvimento gerencial e com toda gama de aprendizagem natural, acidental e não deliberada que ocorre no dia a dia do trabalho. Já a teoria dirige-se a "toda formulação conceitual que descreve, interpreta ou cria essas atividades" (Ibid., p. 5).

Para Fox (1997), como uma área de pesquisa e prática, a aprendizagem gerencial procura suprimir a lacuna deixada pela visão tradicional que concebe a aprendizagem dos executivos apenas através da educação e desenvolvimento gerencial, cujas ênfases estão, respectivamente, na teoria e na prática.

Segundo este autor, o escopo da aprendizagem gerencial envolve três domínios: o mundo da prática gerencial e os mundos da educação e do desenvolvimento gerencial. Como proposto na Figura 1, a aprendizagem gerencial como área de pesquisa e prática é uma interface entre a realidade concreta vivenciada pelo executivo no dia a dia de trabalho e as atividades de desenvolvimento e educação gerencial que estão à sua disposição para suprir suas necessidades de aprendizagem. 
ORGANIZAÇÕES - APREDIZAGEM GERENCIAL: TEORIA E PRÁTICA

Liege Viviane dos S. de Moraes - Maria Aparecida da Silva - Cristiano J. A. C. Cunha

A principal noção que a Figura 1 procura transmitir é que a aprendizagem gerencial não ocorre apenas através do desenvolvimento ou da educação gerencial, mas também no dia a dia do trabalho e, por isso, pesquisa e prática nesta área devem envolver os três domínios apresentados.

\section{Figura 1: O Escopo de Estudo da Aprendizagem Gerencial}

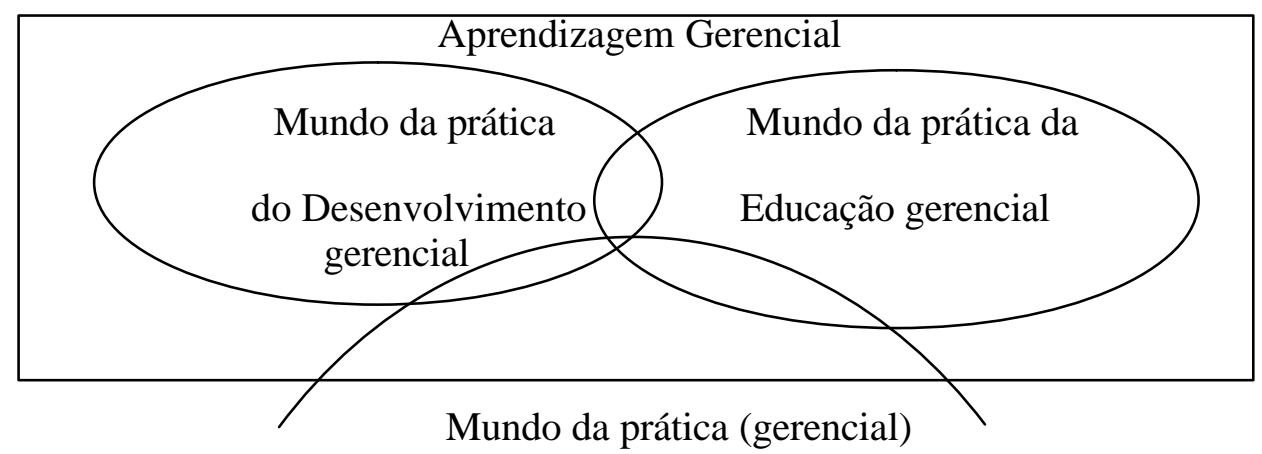

Fonte: Fox (1997)

Esta noção consolidoutse através de uma série de pesquisas empíricas interessadas no processo de aprendizagem gerencial. A próxima seção apresenta alguns comentários sobre o escopo e as descobertas destas investigações.

\section{As pesquisas empíricas internacionais sobre a aprendizagem gerencial}

Embora influenciadas por diferentes perspectivas teóricas e abordagens metodológicas, as investigações sobre aprendizagem gerencial trazem descobertas similares e reveladoras sobre o processo pelo qual os executivos aprendem.

Uma das primeiras pesquisas na área foi realizada por Burgoyne e Hodgson (1983), que analisaram trinta e sete episódios de aprendizagem do dia a dia de trabalho de oito gerentes. A abordagem de pesquisa utilizada foi a fenomenologia com foco na "aprendizagem natural dos gerentes, isso é, a aprendizagem que acontece fora de ambientes de ensino" (p.391). Como resultado, esses autores descobriram três níveis de aprendizagem: aquela decorrente da assimilação de uma informação ou dado para aplicação imediata; a que advêm da transferência de um conhecimento obtido em uma experiência passada e aquela que ocorre quando o gerente "torna-se consciente sobre suas concepções do mundo em geral, como são formadas, ou como ele pode mudá-las" (p.395).

Davies e Easterby-Smith (1984) realizaram um estudo sobre a aprendizagem de 60 gerentes com preocupação de saber se "de fato os gerentes aprendem e se desenvolvem tanto quanto poderiam através de suas experiências diárias de trabalho" (p.169). Este estudo utilizou uma perspectiva de longo prazo sobre a aprendizagem, mas ateve-se apenas àquela que ocorria através da experiência. Descobriram uma relação entre o ambiente de negócio da organização e a aprendizagem e $o$ desenvolvimento gerencial. Concluíram que a experiência de trabalho "é a chave para o desenvolvimento de gerentes" (p. 180) e que os executivos "se desenvolvem primariamente por meio de confrontos com situações e problemas novos em que o repertório de comportamentos deles é inadequado e onde eles têm que desenvolver novas maneiras de tratar essas situações" (p. 180).

Preocupado com a seriedade no desenvolvimento do talento gerencial, Zemke (1985) descreveu um estudo realizado pelo departamento de recursos humanos da Honeywell Corp. Tal estudo objetivava 
ORGANIZAÇÕES - APREDIZAGEM GERENCIAL: TEORIA E PRÁTICA

Liege Viviane dos S. de Moraes - Maria Aparecida da Silva - Cristiano J. A. C. Cunha

diminuir as "inadequações nos programas de desenvolvimento gerencial da empresa e de delinear novas abordagens para o abastecimento de novos gerentes" (p.46). Representando uma revelação inédita na época, este estudo constatou que os executivos aprendiam pelas experiências de trabalho, relacionamentos e treinamentos numa proporção, respectivamente, de 50\%, 30\% e $20 \%$ (Zemke, 1985).

De forma similar, Lowy, Kelleher e Finestone (1986) investigaram a aprendizagem de gerentes de sucesso e revelaram que tal processo é resultado da combinação de características do indivíduo na realização de certas tarefas, em contextos particulares. Constataram que em sua maior parte, a aprendizagem dos gerentes ocorria informalmente, por meio das experiências vivenciadas no trabalho. Tanto que associaram o grau de aprendizagem ao tipo de experiências vivenciadas pelos gerentes no trabalho.

Fundamentada na Teoria Transformativa (Mezirow, 1991), La Paro (1991) conduziu o estudo empírico com 20 gerentes médios de uma organização do setor da saúde, buscando compreender como e o que eles aprendem. Verificou que a aprendizagem era guiada pelas experiências de trabalho que ofereciam novidades, adversidades, diversidades e responsabilidades. Recursos formais e informais foram relatados, mas nenhum gerente considerou as atividades de treinamento e educação como formas significativas de aprendizagem.

A aprendizagem através de relacionamentos foi considerada a mais importante, pois provinham os executivos de informações, feedback, conselhos, críticas e suporte para a ação. La Paro identificou ainda formas emergentes de aprendizagem, como: reflexão, tentativa e erro e observação. Verificou que "o conteúdo de aprendizagem consistia em mais do que apre ndizagem instrumental" (La Paro, 1991, p. 177), ou seja, de conhecimentos técnicos.

De forma parecida, Akin (1993) também investigou como e o que aprenderam 60 gerentes de sucesso de organizações hospitalares. Seu objetivo era contribuir para a construção de teorias sobre a aprendizagem gerencial, já que "teorias e modelos da prática gerencial são bastante elaborados, enquanto teorias da aprendizagem gerencial são em sua maioria simplistas" (p. 161).

Grande parte dos gerentes relacionou suas experiências de aprendizagem à observação de mentores pessoas possuidoras de uma visão de mundo coerente e bem desenvolvida. Outras formas identificadas foram: a adoção de modelos; solução de problemas; a validação e antecipação, e a aprendizagem científica.

Akin (1993) identificou que os executivos aprendiam: habilidades interpessoais; habilidades analíticas e de comunicação; sobre o trabalho, as normas organizacionais e profissionais. Além disso, o autor destaca que os executivos pesquisados obtiam autoconfiança.

Em sua tese de doutorado, Ahmad (1994) buscou identificar os padrões de aprendizagem utilizados por nove diretores de universidades americanas. Utilizou como base a nomenclatura desenvolvida por Cervero, Miller e Dimmock (1986). Ahmad (1994) investigou também o contexto cultural relacionado aos padrões de aprendizagem. Como resultado, constatou que a maior parte da aprendizagem dos diretores pesquisados ocorreu através de atividades informais, como os atos de imitar os outros, de refletir, de tentativa e erro, de fazer o trabalho e de observar.

Embora não seja objetivo deste artigo a discussão de conceitos, é preciso destacar que as atividade informais citadas acima, que não são deliberadas ou planejadas pelo executivo - como refletir e 
ORGANIZAÇÕES - APREDIZAGEM GERENCIAL: TEORIA E PRÁTICA

Liege Viviane dos S. de Moraes - Maria Aparecida da Silva - Cristiano J. A. C. Cunha

relacionar-se - vêm sendo caracterizadas por autoras como Marsick e Watkins (1997), como aprendizagem acidental para definir a natureza não premeditada desta aprendizagem.

Com base num estudo realizado pelo Center for Creative Leadership, Daudelin (1996) conduziu uma pesquisa com 48 executivos de grandes empresas, procurando responder qual tipo de reflexão é mais efetivo para ajudar os executivos a aumentar sua aprendizagem a partir das experiências de trabalho. $\mathrm{O}$ objetivo era diminuir a lacuna entre o "entendimento da natureza da reflexão e nossa habilidade de recomendar ferramentas para aumentar a aprendizagem gerencial" (p.43). Tal estudo ressaltou o potencial de aprendizagem existente nas experiências, sugerindo que se os executivos "despendessem apenas uma hora de reflexão sobre aspectos de uma situação desafiante [...] poderiam aumentar significativamente a aprendizagem daquela situação" (p. 45).

Por outro lado, explorando a transferência de conhecimentos e habilidades dentro das organizações, dos peritos para os novatos, Gherardi, Nicolini e Odella (1998) realizaram um estudo de caso com um gerente de uma empresa italiana de construção civil. Por meio da observação de campo e de perguntas semi-estruturadas, procuraram explorar as diferentes maneiras pelas quais certas habilidades práticas e tácitas eram transferidas dentro de comunidades de prática. Concluíram que a linguagem foi o principal meio para tornar-se competente nas tarefas do dia a dia e que a competência é definida tacitamente pelas relações de poder que só podem ser modificadas pela comunicação.

Por sua vez, Richter (1999) realizou uma pesquisa com sete executivos seniors de diferentes segmentos para explorar a existência de comunidades de prática. Constatou que os executivos faziam parte de comunidades de prática nas quais ocorriam grande parte de sua aprendizagem, de forma não intencional.

Entre os estudos sobre aprendizagem profissional genérica das pessoas nas organizações, ou seja, não especificamente a aprendizagem de executivos, destacam-se os trabalhos de Cervero, Miller e Dimmock, (1996) e de Rigano e Edwards (1998). Eles têm dado suporte às investigações sobre a aprendizagem gerencial e demonstram o crescente interesse em compreender a aprendizagem nas organizações.

Cervero, Miller e Dimmock (1986) realizaram um survey com profissionais de engenharia, constatando que os métodos informais de aprendizagem são mais utilizados pelos profissionais do que os métodos formais.

Já o estudo de Rigano e Edwards (1998) fazia parte de um programa de autodesenvolvimento de uma empresa, cujos objetivos eram: acessar conhecimentos práticos pessoais; melhorar a performance através da ação e reflexão; encorajar novas formas de estratégias de pensamento no ambiente de trabalho. Utilizando como base de pesquisa a hermenêutica e a fenomenologia, estes pesquisadores demonstraram como um engenheiro inseria a reflexão na prática do trabalho diário como forma de aumentar sua performance. A próxima seção expõe comentários de estudos sobre a aprendizagem de executivos brasileiros.

\section{Pesquisas empíricas nacionais sobre a aprendizagem gerencial}

Como exposto anteriormente, as investigações na área da aprendizagem gerencial são incipientes no Brasil. Entre as pesquisas realizadas, destacam-se aquelas empreendidas por um grupo de pesquisadores do Programa de Pós-Graduação em Engenharia de Produção/PPGEP da Universidade Federal de Santa Catarina/UFSC (Silva, 2000; Moraes, 2000; Gottwald, 2001; Lucena, 2001). 
ORGANIZAÇÕES - APREDIZAGEM GERENCIAL: TEORIA E PRÁTICA

Liege Viviane dos S. de Moraes - Maria Aparecida da Silva - Cristiano J. A. C. Cunha

Sendo parte de um mesmo grupo de pesquisa, a abordagem metodológica utilizada por eles tem características em comum. Entre elas, a influência do paradigma interpretativista na construção da pesquisa. Sob esta influência, estes pesquisadores buscaram interpretar e compreender o fenômeno da aprendizagem gerencial através da perspectiva dos sujeitos, seguindo as orientações da pesquisa qualitativa na condução de seus estudos de caso (Merriam, 1998).

Estes estudos adotaram recursos metodológicos da Grounded Theory para a análise e categorização dos dados. Esta abordagem metodológica visa a construção de teorias em pesquisa qualitativa. Assim, utilizaram-se os métodos de comparação constante e de codificação dos dados para captar a essência dos fenômenos estudados dando sentido aos dados (Strauss e Corbin, 1990).

Silva (2000) foi quem deu início às pesquisas sobre aprendizagem gerencial no PPGEP. Interessada primeiramente no processo de mudança organizacional e de aprendizagem organizacional, a autora 'viu' na aprendizagem gerencial um passo anterior, ou seja, o start dos processos em que estava inicialmente interessada. Assim, certa do papel dos executivos na ação organizacional, realizou o estudo empírico de sua tese de doutorado sobre aprendizagem de professores que se tornaram dirigentes universitários.

Sua pesquisa procurou responder: como os diretores aprendem a exercer suas atividades administrativas; o que aprendem ao se tornarem diretores e como as culturas do meio acadêmico influenciam o processo de aprendizagem. Tendo entrevistado os onze diretores das Unidades Universitárias da UFSC, descobriu que eles aprendiam já no exercício do cargo, sob a influência de suas experiências passadas e da cultura acadêmica da unidade que administravam. Constatou que eles autodirecionavam sua aprendizagem através de recursos informais e que havia uma ênfase no desenvolvimento de habilidades voltadas para a prática e para o conhecimento pessoal, bem como de habilidades referentes à administração do tempo, trabalho em equipe e relacionamentos (Silva, 2001, 2002).

Buscando aprofundar os conhecimento na área, a dissertação de mestrado de Moraes (2000) deu continuidade ao estudo de Silva (2000) procurando explorar a agenda de pesquisa proposta por Burgoyne e Hodgson (1983) e Richter (1998) relativa as questões da reflexão e dos relacionamentos no processo de aprendizagem. O objetivo geral deste estudo foi compreender o processo de aprendizagem dos executivos de uma organização hospitalar diante dos desafios diários do trabalho. A pesquisa empírica foi realizada em um hospital de excelência de Porto Alegre/RS es através da amostragem intencional, cinco executivos foram entrevistados.

O estudo concluiu que a aprendizagem gerencial não pode ser classificada apenas como formal e informal, pois não ocorre apenas em momentos isolados e planejados. Verificourse que a aprendizagem ocorria também no dia a dia do trabalho de forma não planejada, através dos relacionamentos que se estabeleceram na trajetória de participação dos gerentes. Uma descoberta relevante de Moraes (2000) é que as mudanças de consciência que se originaram da reflexão crítica sobre a prática destes profissionais, levou os executivos à aprendizagem. Por fim, esta autora identificou que a aprendizagem não se limita a aquisição de habilidades analíticas, mas inclui a aprendizagem sobre si mesmos, sobre a organização e sobre o ambiente externo da mesma.

Em sua dissertação de mestrado, Gottwald (2001) desenvolveu um estudo com cinco executivos do setor automobilístico, procurando compreender o processo de aprendizagem destes sujeitos. Como a empresa pesquisada era uma multinacional instalada no Brasil, esta pesquisadora buscou compreender como os executivos aprendiam a cultura da organização. Como resultado, esta autora concluiu que os 
ORGANIZAÇÕES - APREDIZAGEM GERENCIAL: TEORIA E PRÁTICA

Liege Viviane dos S. de Moraes - Maria Aparecida da Silva - Cristiano J. A. C. Cunha

contextos que mais propiciavam o aprendizado eram aqueles que envolviam novas responsabilidades, bem como o confronto com um contexto organizacional desconhecido. Sobre a aprendizagem da cultura organizacional, constatou que ela era aprendida, principalmente, através da oportunidade da vivência na matriz, sendo que a falta do domínio do idioma alemão é um fator que prejudica muito a aprendizagem gerencial.

Lucena (2001) realizou a pesquisa empírica de seu doutorado sobre a aprendizagem profissional de gerentes-proprietários do setor de varejo de vestuário de Florianópolis/SC. Seu estudo levou à conclusão de que os fatores que motivavam a aprendizagem dos sujeitos eram as demandas no trabalho, causalidades e recompensas. Verificou que a aprendizagem ocorria em grande parte por meio de relacionamentos sociais e se baseava mais no desempenho e na reflexão das atividades de trabalho do que em treinamento e educação. $\mathrm{O}$ conteúdo de aprendizagem dos executivos pesquisados estava relacionado com a organização e com o autoconhecimento.

Demonstrando o interesse crescente de pesquisadores brasileiros sobre a aprendizagem nas organizações, Loiola e Rocha (2002) realizaram um estudo de caso qualitativo que investigou os determinantes da aprendizagem dos indivíduos no processo de seleção de ferramenta CASE para o Estado da Bahia. A pesquisa foi realizada com nove dos dez membros da comissão do processo citado, os quais são funcionários de diversas instituições governamentais da Bahia. As principais conclusões deste estudo referem-se à descoberta de que a aprendizagem nas organizações é um processo tanto intra como interpsíquico, que depende da comunicação e onde a interação social é lugar para a negociação de significados.

\section{CONSIDERAÇÕES FINAIS}

A aprendizagem vista como transferência de conhecimento pode ser equacionada como o ato de comer, onde o conhecimento é a comida e o aprendiz é o catalisador que deve ingerir o tipo de certo de alimento para obter um resultado esperado. Nesta concepção, educação e treinamento são considerados formas de instrução específicas e orientadas para certos objetivos, provendo os profissionais com conhecimentos e habilidades para que possam desempenhar seus papéis.

Essa perspectiva é simplista para que se possa compreender como os adultos aprendem, em geral, e nas organizações, de forma específica. Essa visão passou a ser questionada na medida em que se percebeu as deficiências das práticas tradicionais de educação e desenvolvimento profissional.

A consolidação da aprendizagem gerencial como uma área de pesquisa e prática no campo das organizações está relacionada, principalmente, com a necessidade de criar-se sistemas mais eficientes e responsáveis na árdua tarefa de ajudar os executivos a aprender, de modo a que eles possam lidar com os desafios diários de seus trabalhos.

Por outro lado, a amadurescimento deste campo de estudos também deve-se ao desenvolvimento de novos conceitos na área da educação e aprendizagem de adultos, os quais possibilitam uma releitura do conceito tradicional da aprendizagem gerencial.

Conhecer as recentes descobertas empíricas desta área é extremamente relevante tendo em vista a recente difusão, no Brasil, dos programas de educação para executivos, como os mestrados executivos e os MBA. Esses programas tornaram-se sinônimo de aprendizagem gerencial no país, mas não refletem um esforço proporcional em pesquisas que busquem compreender como os executivos de fato aprendem. 
A compreensão do processo pelo qual os executivos aprendem têm possibilitado a utilização de ferramentas mais adequadas ao ato de ensinar a gestão, facilitando a absorção e aplicação, por parte dos alunos, dos conhecimentos que os programas de educação e desenvolvimento gerencial procuram transmitir.

Além disso, compreender como os executivos aprendem potencializa a utilização, por parte destes profissionais, de recursos de aprendizagem situados no dia a dia do trabalho gerencial. Estes recursos de aprendizagem são extremamente úteis para o desenvolvimento de competências gerenciais e, por serem inerentes ao gerente, não necessitam necessariamente ocorrer através de programa s formação gerencial. A aprendizagem gerencial não é apenas um produto, decorrente da transferência do conhecimento em ocasiões planejadas e isoladas. Aprendizagem gerencial é um processo que ocorre ao longo da trajetória profissional, que deriva e depende de um contexto social particular.

As diversas pesquisas na área indicam a existência de padrões na aprendizagem dos executivos, independentemente de serem brasileiros ou não. A existência destes padrões sugere que este processo é definido, em grande parte, pela característica inerente a todos os executivos, independente de sua nacionalidade: o fato de serem adultos, profissionais que compartilham uma forma de ação particular.

As diferentes pesquisas na área demonstram que os relacionamentos são uma peça fundamental para o processo de aprendizagem gerencial. Grande parte do conhecimento necessário para a prática da gestão encontra-se disseminado entre os membros da organização de forma tácita e vão sendo abstraídos através da trajetória de participação do executivo nesta prática.

Da mesma forma, a reflexão aparece como uma forma significativa para a aprendizagem gerencial em diferentes pesquisas, já que o executivo é um adulto e, como tal, a natureza de sua aprendizagem é emancipatória, ou seja, possui uma dimensão que lhe permite questionar os pressupostos que guiam e restringem sua ação profissional.

A análise mais detalhada dos referenciais apresentados neste artigo demonstra que o que diferencia o processo de aprendizagem dos executivos, no Brasil e no exterior, são aspectos relacionados às características do ambiente em que suas organizações atuam, por exemplo a competitividade do setor. Além disso, a cultura organizacional também influencia a aprendizagem dos executivos na medida que ela pode facilitá- la ou dificultá-la, inib́rla ou incentivá-la.

\section{Notas:}

${ }^{1}$ Utiliza-se como base o conceito de executivo de Mintzberg (1973), para quem este profissional é a pessoa encarregada pela administração "de uma organização ou uma de suas unidades. Assim, presidentes, bispos, chefes de uma seção e técnicos de futebol seriam exemplos de executivo pois a autoridade formal leva à uma posição especial de status na organização" (p. 56).

2 Para explorar mais este conceito ver Lave e Wenger (1991), Richter (1998), Gherardi, Nicolini e Odella (1998) e Antonello e Ruas (2002).

${ }^{3}$ Utiliza-se como base do conceito descrito por Brookfield (1990), que define redes de aprendizagem como atividades informais que resultam da reunião de grupos de pessoas com o mesmo nível de status, que se agregam para trocar informações, idéias, habilidades e conhecimentos.

\footnotetext{
${ }^{4}$ Baseado em Habermas, Mezirow (1981) propõe a existência de três domínios da aprendizagem na fase adulta, os quais estão relacionados com áreas de interesse cognitivo. O primeiro domínio - aprendizagem instrumental - indica o interesse pelo trabalho relacionando-se à solução de problemas e aos 'como fazer'. O segundo - aprendizagem comunicativa relaciona-se com o interesse prático; refere-se às normas consensuais que definem expectativas recíprocas sobre 
ORGANIZAÇÕES - APREDIZAGEM GERENCIAL: TEORIA E PRÁTICA

Liege Viviane dos S. de Moraes - Maria Aparecida da Silva - Cristiano J. A. C. Cunha

comportamento. O terceiro - aprendizagem autoreflexiva ou emancipatória - envolve o interesse pelo autoconhecimento e pelas restrições psicológicas e culturais que influenciam a forma pela qual o indivíduo vê a si próprio.

\section{Artigo recehido em 27.02.2003. Aprovado em 18.08.2003}

\section{REFERÊNCIAS BIBLIOGRÁFICAS}

AKIN, G. Varieties of managerial learning. Health Administration Education. v. 11, n.2, p. 161- 177, 1993.

ANTONELLO, C.S.; RUAS, R. Formação gerencial: pós-grauação Lato Sensu e o papel das comunidades de prática. XXVI ENANPAD, Salvador; Bahia, 2002.

ARGYRIS, C. Double loop learning in organizations. Harvard Business Review. v, n., p. 115-125, setoct, 1977.

BROWN, J. S.; COLLINS, A. e DOUGUID, P. Situated cognition and the culture of earning. Education Researcher. v. 18, n. 1, p. 32-42, 1989.

BROOKFIELD, S. D. Understanding and facilitating adult learning. San Francisco (EUA): JosseyBass Publishers, 1a. Ed, 5a. reimpressão, 1990.

BURGOYNE, J.; REYNOLDS, M. Management learning: integrating perspectives in theory and practice. London: Sage Publications, 1997.

BURGOYNE, J. HODGSON, V. Natural learning and managerial action: a phenomenological study in the field setting. Journal of Management Studies. v. 20, n. 3, 1983.

CANDY, P. Self direction for lifelong learning: a comprehensive guide to theory and practice. San Francisco (USA): Jossey-Bass, 1991.

CARNEVALE, A. P. The learning enterprise. Training and Development Journal. v. 40 n. 1, p. 18-26, 1986.

CRANTON, P. Professional development as transformative learning: new perspectives for teachers of adults. San Francisco (USA): Jossey-Bass Publishers, 1996.

CERVERO, R. M.; MILLER J. D.; DIMMOCK, K. H. The formal \& informal learning activities of practicing engineers. Engineering Education. November, p. 112-114, 1986.

DAVIES, J.; EASTERBY-SMITH, M. Learning and developing from managerial work experience. Journal of Management Studies. v. 21, n. 2, p. 169-183, 1984.

DAUDELIN, M. Learning from experience through reflection. Organization Dynamics. Winter, p. 3648, 1996.

EISENHART, M. Learning as movement in networks of knowledge and power. Educational Researcher. v. 24, n. 7, p. 35-36, 1995. 
FOX, S. From management education and development to the study of management learning. IN: BURGOYNE, John and REYNOLDS, M.. Management Learning: Integrating Perspectives in Theory and Practice. Sage Publications, London, 1997, p. 21-37.

FREIRE, P. Educação como prática da liberdade. 13ªed. Rio de Janeiro: Paz e Terra, 1982.

GHERARDI, S.; NICOLINI, D.; ODELLA, F. Toward a social understanding of how people learn in organizations. Management Learning. v. 29, n. 3, p. 273-297, 1998.

GOTTWALD, G. A aprendizagem de executivos no setor automobilístico - estudo de caso na Volkswagen /Audi do Brasil. 2001. Dissertação (mestrado em Engenharia de Produção) - Centro tecnológico, Universidade Federal de Santa Catarina.

KOLB, D. Management and the learning process. California Management Review. v. 18, n. 3, p. 21-31, 1976.

MUMFORD, Alan. Action learning as a vehicle for learning. In: MUMFORD, Alan. Action learning at work. Hampshire: Gower Publishing Limited, 1997.

LA PARO, M. E. Health care middle managers: what and how they learn. New York: Columbia University, 1991. Tese de Doutorado.

LAVE, J.; WENGER, E. Situated learning: legitimate peripheral participation. Cambridge: Cambridge University Press, 1991.

LIVINGSTON, J. The myth of the well-educated manager. Harvard Business Review. 1971, v. 49, n. 1, p. 79-89.

LOIOLA, E.; ROCHA, M. C. F. Aprendizagem no Processo de Seleção de Ferramenta CASE para o Estado da Bahia. Revista de Administração Contemporânea. V.6, n.2, 2002.

LOWY, A.; KELLEHER, D.; FIRESTONE, P. Management learning: beyond program design. Training and Development Journal, 1986, jun, p. 34-37.

LUCENA, E. A. A aprendizagem profissional de gerentes-proprietario do setor de varejo de vestuário de Florianópolis. 2001. 165 .Tese (Doutorado em Engenharia de Produção) - Centro tecnológico, Universidade Federal de Santa Catarina.

MARSICK, V.; WATKINS, K. Lessons from informal and incidental learning. In: Burgoyne, J.; Reynolds, M. Management learning: integrating perspectives in theory and practice. London: Sage Publications, 1997.

MARSICK, V. Learning in the workplace: the case for reflectivity and critical reflectivity. Adult Education Quarterly. v.38, n. 4, Summer, p. 187-198, 1988.

MERRIAM, S. Qualitative research and case study applications in Education. San Francisco: JosseyBass, 1998. 
MERRIAM, S.; CAFFARELLA, R. Learning in adulthood: a comprehensive guide. San Francisco: Jossey-Bass, 1991.

MEZIROW, J. Transformative dimensions of adult learning. San Francisco (USA): Jossey-Bass, 1991.

MEZIROW, J. Contemporary paradigms of learning. Adult Education Quartely. v. 46, n. 3, spring, p. 158-173, 1996.

MEZIROW, J. A critical theory of adult learning. Adult Education, 32:3-24, 1981.

MINTZBERG, Henry. The nature of managerial work. New York: Harper Collins Publishers, 1973.

MORAES, L. V. S. A dinâmica da aprendizagem gerencial: o caso do Hospital Moinhos de Vento. 2000. Dissertação (Mestrado em Engenharia de Produção) - Centro tecnológico, Universidade Federal de Santa Catarina.

MUNFORD, Alan. Action learning as a vehicle for learning. In: MUMFORD, Alan. Action learning at work. Hampshire: Gower Publishing Limited, 1997.

REYNOLDS, M. Reflection and critical reflection in management learning. Management Learning. v. 29, n. 2, p. 183-200, 1998.

RICHTER, I. Individual and organizational learning at the executive level. Management Learning. v. 29, n. 3, p. 299-316, 1998.

RICHTER, I. Executive learning and organizational learning: diffuse power or dormant potential. In: I Congress of Organizational Learning., June, 1999, Lancaster. Acesso em out/99. Disponível em: <http://notes.lancs.ac.uk/pub/ol3.nsf>

RIGANO, D.; EDWARDS, J. Incorporating reflection into work practice. Management Learning. v. 29, n. 4, p. 431-446, 1998.

RUAS, R. Mestrado executivo, formação gerencial e a noção de competências: provocações $e$ desafios. XXVI ENANPAD, Salvador, Bahia, 2002.

RUAS, Roberto. Mestrado executivo, formação gerencial e a noção de competências: provocações e desafios. XXV ENANPAD, Campinas; São Paulo, 2001 (a).

RUAS, R. Desenvolvimento de competências gerenciais e contribuição da aprendizagem organizacional. In: FLEURY, M. T., OLIVEIRA, M. M. Gestão estratégica do conhecimento: Integrando aprendizagem, conhecimento e competências. São Paulo: Atlas, 2001 (b).

SCHÖN, D. The reflective practitioner: how professionals think in action. London: Temple Smith, 1983.

SILVA, M. A. O que os professores da Universidade Federal de Santa Catarina aprenderam para dirigir as unidades universitárias. XXV ENANPAD, Campinas, são Paulo, 2001. 
ORGANIZAÇÕES - APREDIZAGEM GERENCIAL: TEORIA E PRÁTICA

Liege Viviane dos S. de Moraes - Maria Aparecida da Silva - Cristiano J. A. C. Cunha

SILVA, M. A., MORAES, L. V. S. Como os professores da universidade federal de santa catarina aprenderam a dirigir as unidades universitárias. XXVI ENANPAD, Salvador, Bahia, 2002.

SILVA, M. A. A aprendizagem de professores da Universidade Federal de Santa Catarina para dirigir as unidades universitárias. Florianópolis: Universidade Federal de Santa Catarina 2000. Tese de Doutorado.

STRAUSS, A.; CORBIN, J. Basics of Qualitative Research: Grounded Theory procedures and techniques. London: Sage, 1990.

TOUGH, A. The adult's learning projects: a fresh approach to theory and practice in adult learning. $2^{\mathrm{a}}$ edição. Ontario: Ontario Institute for Studies in Education, 1971.

ZEMKE, R. The Honeywell Studies: how managers learn to manage? Training, v. 22, n. 8, p. 46-51, 1985.

\section{Liege Viviane dos Santos de Moraes}

Doutoranda no Programa de Pós-Graduação em Engenharia de Produção (PPGEP/UFSC). Mestre em Engenharia de Produção (PPGEP/UFSC)

E-mail: liege75@aol.com

Endereço: UFSC/PPGEP, Sala 26 - Escaninho 13 Campus Universitário - Trindade - Caixa Postal 476

Florianópolis - SC, 88040-900.

Interesses de pesquisa: Aprendizagem gerencial, cultura organizacional.

\section{Maria Aparecida da Silva}

Professora na UDESC - Mestrado em Administração.Doutorado em Engenharia de Produção

E-mail: maria_aparecida@joinville.udesc.br

Endereço: Av. Prof. Avelino Marcante s/n. Campus Universitário, Joinville - SC, 89223130.

Interesses de pesquisa: Aprendizagem gerencial, cultura organizacional.

\section{Cristiano J. C. A. Cunha}

Professor do Programa de Pós-Graduação em Engenharia de Produção (PPGEP/UFSC)

E-mail: cunha@iea.com.br

Endereço: UFSC/PPGEP, Sala 26 - Escaninho 13 Campus Universitário - Trindade - Caixa Postal 476 Florianópolis - SC, 88040-900.

Interesses de pesquisa: Aprendizagem gerencial, planejamento estratégico. 